\title{
Cooperative localization based on severely quantized RSS measurements in wireless sensor network
}

Di Jin, Feng Yin, Carsten Fritsche, Abdelhak M. Zoubir and Fredrik Gustafsson

\author{
Linköping University Post Print
}

\section{Tweet}

N.B.: When citing this work, cite the original article.

Original Publication:

Di Jin, Feng Yin, Carsten Fritsche, Abdelhak M. Zoubir and Fredrik Gustafsson, Cooperative localization based on severely quantized RSS measurements in wireless sensor network, 2016, IEEE International Conference on Acoustics, Speech and Signal Processing (ICASSP), (), , 4214-4218.

http://dx.doi.org/10.1109/ICASSP.2016.7472471

Copyright: http://www.ieee.org

Postprint available at: Linköping University Electronic Press

http://urn.kb.se/resolve?urn=urn:nbn:se:liu:diva-128632 


\title{
COOPERATIVE LOCALIZATION BASED ON SEVERELY QUANTIZED RSS MEASUREMENTS IN WIRELESS SENSOR NETWORK
}

\author{
Di Jin* ${ }^{*}$ Feng Yin ${ }^{* \dagger}$, Carsten Fritsche ${ }^{\ddagger}$, Abdelhak M. Zoubir ${ }^{*}$, and Fredrik Gustafsson ${ }^{\ddagger}$ \\ * Signal Processing Group \\ Technische Universität Darmstadt \\ Darmstadt, Germany \\ \{djin, zoubir\}@spg.tu-darmstadt.de \\ ${ }^{* \dagger}$ Ericsson AB \\ Linköping, Sweden \\ feng.yin@ericsson.com \\ $\ddagger$ Division of Automatic Control
Linköping University
Linköping, Sweden
$\{$ carsten, fredrik\}@isy.liu.se
}

\begin{abstract}
We study severely quantized received signal strength (RSS)based cooperative localization in wireless sensor networks. We adopt the well-known 'sum-product algorithm over a wireless network' (SPAWN) framework in our study. To address the challenge brought by severely quantized measurements, we adopt the principle of importance sampling and design appropriate proposal distributions. Moreover, we propose a parametric SPAWN in order to reduce both the communication overhead and the computational complexity. Experiments with real data corroborate that the proposed algorithms can achieve satisfactory localization accuracy for severely quantized RSS measurements. In particular, the proposed parametric SPAWN outperforms its competitors by far in terms of communication cost. We further demonstrate that knowledge about non-connected sensors can further improve the localization accuracy of the proposed algorithms.
\end{abstract}

Index Terms - Distributed cooperative localization, quantized RSS, SPAWN, wireless sensor network

\section{INTRODUCTION}

Position information is crucial to various wireless sensor network (WSN) applications, where position related measurements, such as time-of-arrival, RSS, and angle-of-arrival, are often severely quantized due to limited sensor readings, storage and bandwidth shortage, etc.

In this paper, we consider distributed cooperative localization based on severely quantized RSS measurements. Among different types of methods as surveyed in [1], the SPAWN algorithm [2] is a promising solution. To reduce computational complexity and communication load, many variants have been built upon it. For instance, [3-6] introduce parametric representation of local belief messages and internal messages and [7] introduces censoring policies into the SPAWN framework. To increase localization accuracy in loopy networks, additional variants of the nonparametric belief propagation (NBP) have been proposed in $[8,9]$, where RSS measurements are first converted into relative distances between sensor nodes and then used in the NBP. To the best of our knowledge, the existing algorithms only consider the case when measurements in the SPAWN or the NBP are relative distances contaminated with additive errors. We focus on the SPAWN and aim to develop SPAWN-type algorithms specifically for severely quantized RSS measurements.

Our contributions are as follows. First, we propose a sampling scheme that is crucial to apply SPAWN-type algorithms to quantized RSS measurements. Second, we develop a parametric SPAWN. Third, we evaluate the proposed algorithms using real data.

The remainder of this paper is organized as follows. Section 2 formulates the problem at hand. In Section 3, we explain in depth several strategies proposed by us in order to solve the quantized RSS-based cooperative localization problem using the SPAWN and its parametric variant. The proposed algorithms are evaluated in Section 4 with real data. Finally, the paper is concluded in Section 5.

\section{PROBLEM FORMULATION}

We consider a WSN with $N$ stationary sensor nodes in a two-dimensional (2-D) space (although extension to 3-D is straightforward). Let $\mathcal{N}_{u}=\left\{1,2, \ldots, N_{u}\right\}$ be the index set of the agents, whose positions are unknown, and let $\mathcal{N}_{a}=$ $\left\{N_{u}+1, N_{u}+2, \ldots, N\right\}$ be the index set of the anchors with known positions. The position of node $i$ is denoted by $\mathbf{x}_{i}=$ $\left[x_{i}, y_{i}\right]^{T}$ and it is modelled stochastically with a prior probability $p_{i}\left(\mathbf{x}_{i}\right)$. Node $i$ can communicate with a subset of sensors, which are called its neighbors and whose index set is denoted by $\mathcal{N}_{\rightarrow i}$. If we have $k \in \mathcal{N}_{\rightarrow j}, k \notin \mathcal{N}_{\rightarrow i}$ and $j \in \mathcal{N}_{\rightarrow i}$, then node $k$ is a 2-hop neighbor of node $i$.

Adopting the commonly used log-distance pathloss model, the continuous-valued RSS, $r_{j i}$, w.r.t. nodes $j$ and $i$, is represented as

$$
r_{j i}=\underbrace{A_{0}-10 n_{p} \log _{10}\left(d_{j i} / d_{0}\right)}_{g_{j i}\left(\mathbf{x}_{i}, \mathbf{x}_{j}\right)}+v_{j i}
$$

where $A_{0}$ denotes the received power at a predefined refer- 
ence distance $d_{0}, n_{p}$ denotes the propagation pathloss exponent, $d_{j i} \triangleq\left\|\mathbf{x}_{i}-\mathbf{x}_{j}\right\|_{2}$ is the Euclidean distance between nodes $j$ and $i$, and the error terms $v_{j i} \sim \mathcal{N}\left(0, \sigma_{j i}^{2}\right), j \in$ $\mathcal{N}_{\rightarrow i}, i=1,2, \ldots, N$, account for the propagation shadowing effect and are assumed to be mutually independent. We assume that the parameters $A_{0}, d_{0}, n_{p}, \sigma_{j i}$ are known.

The measurement $z_{j i}$, measured at sensor $i$, is obtained by quantizing $r_{j i}$ using an $S$-levelled quantization operator $Q(\cdot)$,

$$
z_{j i}=Q\left(r_{j i}\right)=\left\{\begin{array}{cc}
0 & \text { if } P_{0} \leq r_{j i}<P_{1}, \\
1 & \text { if } P_{1} \leq r_{j i}<P_{2}, \\
\vdots & \vdots \\
S-1 & \text { if } P_{S-1} \leq r_{j i}<P_{S}
\end{array}\right.
$$

where $P_{0}, P_{1}, \ldots, P_{S}$ are the quantization levels with $P_{0}=$ $-\infty, P_{S}=+\infty$. The collection of all quantized RSS measurements is denoted by z. In light of Eq. (1) and Eq. (2), it is easy to verify that

$$
\operatorname{Pr}\left(z_{j i}=s \mid \mathbf{x}_{i}, \mathbf{x}_{j}\right)=\Phi\left(\frac{P_{s+1}-g_{j i}}{\sigma_{j i}}\right)-\Phi\left(\frac{P_{s}-g_{j i}}{\sigma_{j i}}\right),
$$

where $\Phi(\cdot)$ is the standard Gaussian cumulative distribution function, $s=0,1, \ldots, S-1$, and $g_{j i}$ is used as a shorthand notation for $g_{j i}\left(\mathbf{x}_{i}, \mathbf{x}_{j}\right)$. Our objective is to estimate the marginal posterior probability density function (pdf) of each agent's position $p\left(\mathbf{x}_{i} \mid \mathbf{z}\right)$, and the corresponding position $\mathbf{x}_{i}$, $\forall i \in \mathcal{N}_{u}$.

\section{COOPERATIVE LOCALIZATION ALGORITHMS}

We adapt two SPAWN-type algorithms to severely quantized RSS data. Given the signal model in Section 2, the internal message as well as the belief message of agent $i$ 's position are updated in the $(\eta+1)$ th iteration as follows:

$$
\begin{aligned}
I_{j i}^{\eta}\left(\mathbf{x}_{i}\right) & =\int \operatorname{Pr}\left(z_{j i} \mid \mathbf{x}_{i}, \mathbf{x}_{j}\right) B_{j}^{\eta}\left(\mathbf{x}_{j}\right) d \mathbf{x}_{j}, \\
B_{i}^{\eta+1}\left(\mathbf{x}_{i}\right) & \propto p_{i}\left(\mathbf{x}_{i}\right) \prod_{j \in \mathcal{N}_{\rightarrow i}} I_{j i}^{\eta}\left(\mathbf{x}_{i}\right),
\end{aligned}
$$

where $B_{j}^{\eta}\left(\mathbf{x}_{j}\right), j \in \mathcal{N}_{\rightarrow i}$ are the old belief messages from agent $i$ 's neighbors, $I_{j i}^{\eta}\left(\mathbf{x}_{i}\right)$ is the internal message in accordance to sensor $j$, and $B_{i}^{\eta+1}\left(\mathbf{x}_{i}\right)$ is the updated belief message of agent $i$ 's position. After a sufficient number of iterations, the belief message $B_{i}\left(\mathbf{x}_{i}\right)$ can be regarded as a good approximation of the marginal posterior pdf $\hat{p}\left(\mathbf{x}_{i} \mid \mathbf{z}\right), \forall i \in \mathcal{N}_{u}$.

\subsection{Particle Based SPAWN}

As suggested by its name, particle based SPAWN algorithm utilizes weighted particles to represent both the internal messages and the belief messages. Due to the use of quantized RSS measurements, we propose a novel way of generating weighted particles for the internal message. Generation of weighted particles $\left\{\mathbf{x}_{i}^{r, \eta+1}, w_{i}^{r, \eta+1}\right\}_{r=1}^{R}$ of the updated belief message $B_{i}^{\eta+1}\left(\mathbf{x}_{i}\right)$ follows the principle of mixture importance sampling given in [10].

Given a set of weighted particles, $\left\{\mathbf{x}_{j}^{r, \eta}, w_{j}^{r, \eta}\right\}_{r=1}^{R}$, of the belief message $B_{j}^{\eta}\left(\mathbf{x}_{j}\right)$ received from neighbor $j$, we wish to generate weighted particles, $\left\{\mathbf{x}_{j i}^{r, \eta}, w_{j i}^{r, \eta}\right\}_{r=1}^{R}$, of the internal message $I_{j i}^{\eta}\left(\mathbf{x}_{i}\right)$ according to the formula given in [10], namely,

$$
\mathbf{x}_{j i}^{r, \eta}=\mathbf{x}_{j}^{r, \eta}+d_{j i}^{r, \eta}\left[\cos \left(\theta^{r, \eta}\right), \sin \left(\theta^{r, \eta}\right)\right]^{T},
$$

where $\left\{d_{j i}^{r, \eta}\right\}_{r=1}^{R}$ is a set of distances drawn from the likelihood function $\operatorname{Pr}\left(z_{j i}=s \mid d_{j i}\right)$, and $\left\{\theta^{r, \eta}\right\}_{r=1}^{R}$ is a set of angles drawn from the uniform distribution $\mathcal{U}[0,2 \pi)$. For notational brevity in the sequel, the iteration index $\eta$ and the subscript $j i$ will be omitted.

Since direct sampling from $\operatorname{Pr}(z=s \mid d)$ is not straightforward, we resort to importance sampling [11]. The profiles of $\operatorname{Pr}(z=s \mid d)$ for different $s$ can be classified into three cases as explained in Fig. 1. Based on this observation, we distinguish these cases and design the proposal distributions for $\operatorname{Pr}(z=s \mid d)$ independently. The dashed lines in Fig. 1 correspond to the proposal distributions in different cases.

In the first and second cases, we propose to construct a proposal distribution using a mixture of a uniform distribution and a triangle distribution. Concretely, the proposal distribution $q(d)$ for the case $s=0$ is given by

$$
\begin{aligned}
q(d) & =\frac{1}{1+C_{a}} \mathcal{U}\left[d_{h}, d_{\text {thres }}\right)+\frac{C_{a}}{1+C_{a}} \Delta\left(d_{l}, d_{h}, d_{l}\right), \\
d_{l} & =d_{0} 10^{\frac{A_{0}-P_{1}-3 \sigma}{10 n_{p}}}, d_{h}=d_{0} 10^{\frac{A_{0}-P_{1}+3 \sigma}{10 n_{p}}}
\end{aligned}
$$

where $C_{a}$ is a scaling factor that makes $q(d)$ continuous, $d_{\text {thres }}$ can be set to the communication range, and $\Delta(a, b, c)$ stands for a triangle distribution and its definition will be given later. While for the case $s=S-1$, we have

$$
\begin{aligned}
q(d) & =\frac{1}{1+C_{a}} \mathcal{U}\left[0, d_{l}\right)+\frac{C_{a}}{1+C_{a}} \Delta\left(d_{l}, d_{h}, d_{l}\right) \\
d_{l} & =d_{0} 10^{\frac{A_{0}-P_{S-1}-3 \sigma}{10 n_{p}}}, d_{h}=d_{0} 10^{\frac{A_{0}-P_{S-1}+3 \sigma}{10 n_{p}}} .
\end{aligned}
$$

The triangle distribution $d \sim \Delta(a, b, c)$ is defined as

$$
p_{\Delta}(d)=\left\{\begin{array}{cl}
\frac{2(d-a)}{(c-a)(b-a)} & \text { if } a \leq d<c \\
\frac{2(b-d)}{(b-c)(b-a)} & \text { if } c \leq d \leq b \\
0 & \text { otherwise }
\end{array}\right.
$$

Both $d_{l}$ and $d_{h}$ are chosen such that $\operatorname{Pr}(z=s \mid d)$ approaches to 1 at $d_{l}$ and to 0 at $d_{h}$. For the third case, i.e., when $s=$ $1,2, \ldots, S-2$, we use a log-normal distribution as the proposal distribution, 


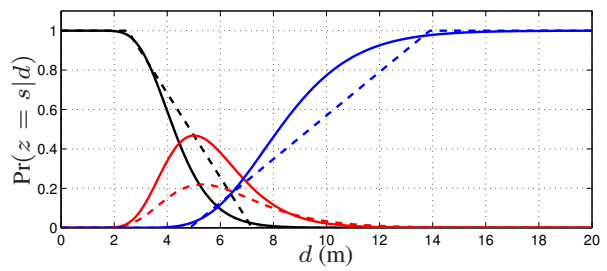

Fig. 1: The likelihood function $\operatorname{Pr}(z=s \mid d)$ and the proposal distribution $q(d)$ are indicated by the solid and dashed lines, respectively. From left to right, the black lines correspond to the case $s=S-1$, the red lines for $s=1,2, \ldots, S-2$, and the blue lines for $s=0$.

$$
\begin{aligned}
& q(d)=\frac{C}{d} \exp \left(-\frac{\left(10 n_{p} \log _{10}\left(\frac{d}{d_{0}}\right)-P_{0}+P_{s}\right)^{2}}{2 \sigma_{\text {new }}^{2}}\right), \\
& \sigma_{\text {new }}^{2}=\sigma^{2}+\frac{\left(P_{s+1}-P_{s}\right)^{2}}{3}, C=\frac{10 n_{p}}{\sqrt{2 \pi} \sigma_{\text {new }} \log (10)},
\end{aligned}
$$

are chosen so that the proposal distribution $q(d)$ matches the target distribution $\operatorname{Pr}(z=s \mid d)$ well.

Up to this point, a distance sample, $d^{r}$, can be generated according to Eq. (6), or Eq. (7) or Eq. (8), depending on the quantized RSS measurement. The weight is simply the ratio of the target distribution and the proposal distribution

$$
w_{d}^{r} \propto \frac{\operatorname{Pr}\left(z=s \mid d^{r}\right)}{q\left(d^{r}\right)},
$$

with $\left\{w_{d}^{r}\right\}_{r=1}^{R}$ that sum up to 1 .

In contrast, generating angle samples from a uniform distribution is fairly simple. Eventually, a particle representation of the internal message can be generated using Eq.(5) and the corresponding weights are simply calculated by

$$
w_{j i}^{r} \propto w_{j}^{r} w_{d}^{r}
$$

where $\left\{w_{j}^{r}\right\}_{r=1}^{R}$ are the weights assigned to $\left\{\mathbf{x}_{j}^{r}\right\}_{r=1}^{R}$. The analytical approximation of the internal message is required in the SPAWN-type algorithms. Based on the weighted particles, $I_{j i}\left(\mathbf{x}_{i}\right)$ is approximated by

$$
I_{j i}\left(\mathbf{x}_{i}\right) \approx \sum_{r=1}^{R} w_{j i}^{r} p_{\mathcal{N}}\left(\mathbf{x}_{i} ; \mathbf{x}_{j i}^{r}, \mathbf{\Sigma}_{j i}\right),
$$

where $p_{\mathcal{N}}\left(\mathbf{x}_{i} ; \mathbf{x}_{j i}^{r}, \boldsymbol{\Sigma}_{j i}\right)$ stands for the pdf of a Gaussian distribution with mean $\mathbf{x}_{j i}^{r}$ and appropriately chosen covariance matrix $\boldsymbol{\Sigma}_{j i}$.

\subsection{Parametric SPAWN}

Our next aim is to design a parametric SPAWN algorithm for severely quantized RSS measurements. Primarily, update of

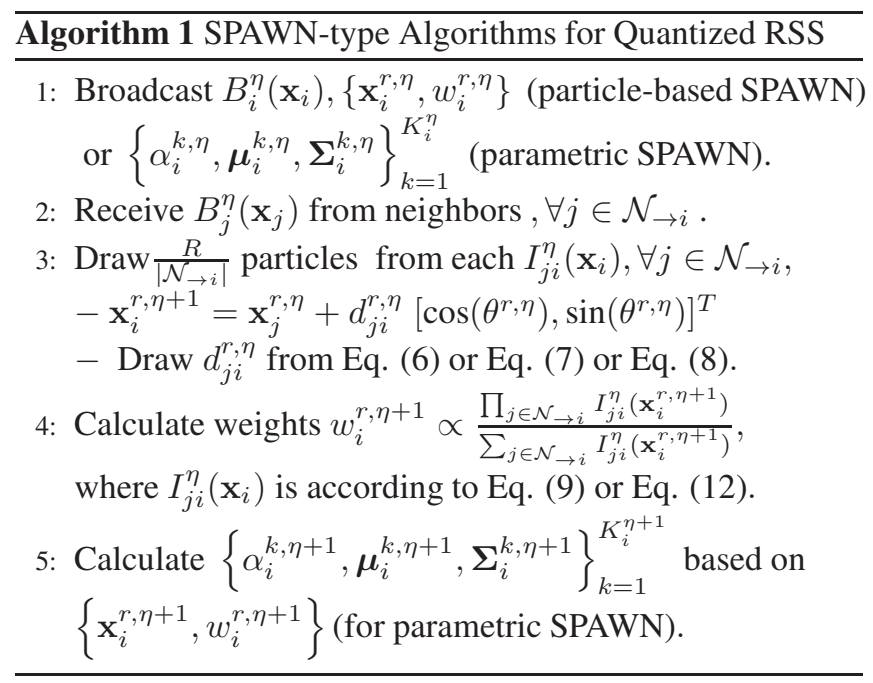

the belief messages still relies on particles. Introducing appropriate parametric models for the belief messages and internal messages is for the sake of reducing communication load and computational complexity, respectively. Similar to our previous work in [12], we approximate the belief message by a finite mode Gaussian mixture model, i.e.,

$$
B_{j}\left(\mathbf{x}_{j}\right) \approx \sum_{k=1}^{K_{j}} \alpha_{j}^{k} p_{\mathcal{N}}\left(\mathbf{x}_{j} ; \boldsymbol{\mu}_{j}^{k}, \mathbf{\Sigma}_{j}^{k}\right)
$$

where $\alpha_{j}^{k}, k=1,2, \ldots, K_{j}$, are the mixture coefficients that sum up to 1 , and $K_{j}$ is the number of Gaussian components. Inserting Eq. (10) into Eq. (4a) gives

$$
I_{j i}\left(\mathbf{x}_{i}\right)=\sum_{k=1}^{K_{j}} \alpha_{j}^{k} \underbrace{\int \operatorname{Pr}\left(z_{j i} \mid \mathbf{x}_{i}, \mathbf{x}_{j}\right) p_{\mathcal{N}}\left(\mathbf{x}_{j} ; \boldsymbol{\mu}_{j}^{k}, \mathbf{\Sigma}_{j}^{k}\right) d \mathbf{x}_{j}}_{G_{j i}^{k}\left(\mathbf{x}_{i}\right)} .
$$

It is apparent from Eq. (11) that each integral $G_{j i}^{k}\left(\mathbf{x}_{i}\right)$ is the convolution of a nonlinear function with a Gaussian density function. We propose to approximate the convolution result by replacing the Gaussian with its mean parameter and by expanding the resulting function appropriately, i.e., the nonlinear function itself with tunable parameters. Therefore, we propose a parametric model $\mathcal{G}\left(\mathbf{x}_{i} ; \boldsymbol{\mu}, \hat{\sigma}\right)$

$\mathcal{G}\left(\mathbf{x}_{i} ; \boldsymbol{\mu}, \hat{\sigma}\right)=\Phi\left(\frac{P_{s+1}-g_{j i}\left(\mathbf{x}_{i}, \boldsymbol{\mu}\right)}{\hat{\sigma}}\right)-\Phi\left(\frac{P_{s}-g_{j i}\left(\mathbf{x}_{i}, \boldsymbol{\mu}\right)}{\hat{\sigma}}\right)$, where $P_{s}, P_{s+1}, A_{0}, n_{p}, d_{0}$ are the parameters of this model, but ignored here for notational brevity. With this model, the convolution result is estimated using

$$
G_{j i}^{k}\left(\mathbf{x}_{i}\right) \approx \mathcal{G}\left(\mathbf{x}_{i} ; \boldsymbol{\mu}_{j}^{k}, \hat{\sigma}_{j i}^{k}\right),
$$

where $\hat{\sigma}_{j i}^{k}=\sqrt{\sigma_{j i}^{2}+\operatorname{Tr}\left(\boldsymbol{\Sigma}_{j}^{k}\right)}$. Finally, the parametric model of the internal message $I_{j i}\left(\mathbf{x}_{i}\right)$ is given by

$$
I_{j i}\left(\mathbf{x}_{i}\right) \approx \sum_{k=1}^{K_{j}} \alpha_{j}^{k} \mathcal{G}\left(\mathbf{x}_{i} ; \boldsymbol{\mu}_{j}^{k}, \hat{\sigma}_{j i}^{k}\right) .
$$




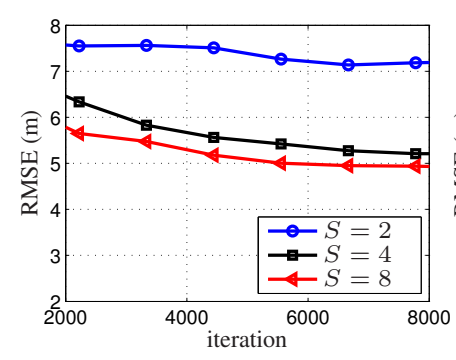

(a)

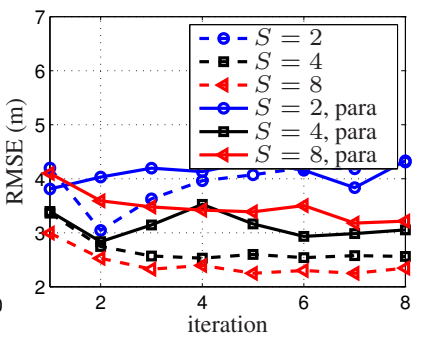

(b)
Fig. 2: RMSE of the distributed MLE in (a) and that of the SPAWNtype algorithms in (b). In (b), the dashed lines and solid lines correspond to the particle based and parametric SPAWN, respectively.

For completeness, the SPAWN-type algorithms are outline in Algorithm 1 w.r.t. node $i$ in $\eta$ th iteration.

\section{EXPERIMENTAL RESULTS}

The proposed algorithms are evaluated using the real sensor network and RSS measurements in [13]. The RSS measurements are uniformly quantized with different quantization levels, $S \in\{2,4,8\}$, giving rise to quantized RSS measurements. The environmental parameters, $A_{0}, n_{p}, d_{0}, \sigma^{2}$ are chosen according to [13]. The distributed maximum likelihood estimator (MLE) from Algorithm 1 in [2] is used as a competitor. For the distributed MLE, the initial position of each agent is randomly chosen over the space. For the SPAWN-type algorithms, $p_{i}\left(\mathbf{x}_{i}\right)$ is simply a uniform distribution. In the SPAWN-type algorithms, $R=1000$ particles are used and the maximum number of Gaussian components is 5 . The SPAWN-type algorithms find the final position estimates using the minimum mean squared error (MMSE) estimator based on the estimated posterior marginal pdfs.

The overall root mean squared errors (RMSEs) of the distributed MLE and the SPAWN-type algorithms over different quantization levels are shown in Fig. 2. To keep the range of $y$ axis in both figures as close as possible, the result of the distributed MLE in the first 2000 iterations are omitted. First, for each quantization level, the proposed algorithms achieve higher localization accuracy than the distributed MLE. In the proposed algorithms, the uncertainty about a position is communicated between neighbors and it contains more information than one point estimate that is shared in the distributed MLE. Second, the proposed algorithms require several iteration steps to achieve a satisfactory localization accuracy; while the distributed MLE needs hundreds. In each iteration, the amount of parameters broadcast by each node in the parametric SPAWN is comparable to that in the distributed MLE. Therefore, the parametric SPAWN requires a profoundly lighter communication load than the distributed MLE. Third, the parametric SPAWN is inferior to the SPAWN in terms

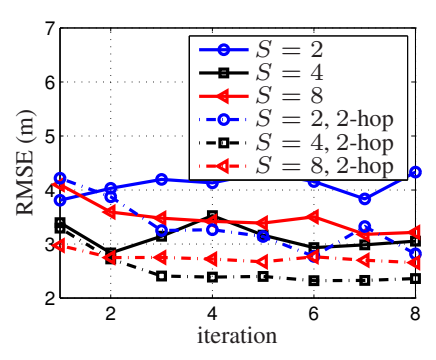

(a)

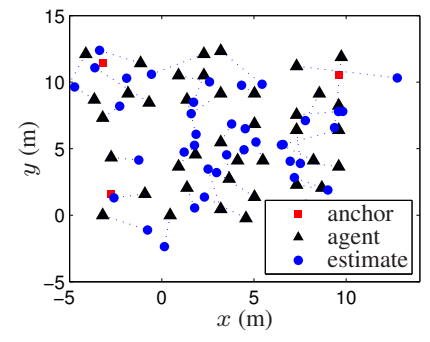

(b)
Fig. 3: The performance of the parametric SPAWN with and without knowledge about 2-hop neighbors: (a) RMSE (b) estimated positions using the parametric SPAWN based on proximity measurements and knowledge about 2-hop neighbors.

of localization accuracy, since the parametric model can not approximate the messages as accurate as the particle-based approximation. Further investigations have shown that both SPAWN-type algorithms do not perform well in the case of $S=2$, since the proximity measurements gives too much freedom to the localization problem.

It is clear that the distance between a node and its 2hop neighbor should be larger than the communication range. Simply speaking, the particles of a node's position located too close to its 2-hop neighbors should be punished. The knowledge about its 2-hop neighbors can be obtained from its neighbors, while at the cost of additional communication load. Next, we investigate the influence of the information from 2-hop neighbors on the localization performance. Due to the large simulation time of the SPAWN, only the parametric SPAWN is considered here. The RMSEs of the parametric SPAWN with and without knowledge about the 2-hop neighbors are depicted in Fig. 3a. It is apparent that the knowledge about 2-hop neighbors improve the localization performance of the parametric SPAWN. Thanks to the additional information from the 2-hop neighbors, more constraints are added to the localization problem and the localization accuracy is improved. Accordingly, for proximity measurements, the position estimate using the parametric SPAWN with additional knowledge about the 2-hop neighbors is illustrated in Fig. 3b. Apparently, based on proximity measurements, the parametric SPAWN provides satisfactory localization accuracy.

\section{CONCLUSION}

We have studied severely quantized RSS-based cooperative localization. To fit quantized RSS measurements in the SPAWN framework, we have proposed novel proposal distributions. Furthermore, we have proposed the parametric SPAWN by designing an appropriate parametric model. Our evaluation results have shown that the proposed algorithms demonstrate satisfactory localization performance. In particular, further knowledge about 2-hop neighbors enhances the localization accuracy of the parametric SPAWN. 


\section{REFERENCES}

[1] F. Yin, C. Fritsche, D. Jin, F. Gustafsson, and A. M. Zoubir, "Cooperative localization in WSNs using Gaussian Mixture Modeling: Distributed ECM algorithms," IEEE Trans. Signal Process., vol. 63, no. 6, pp. 1448-1463, Mar. 2015.

[2] H. Wymeersch, J. Lien, and M. Z. Win, "Cooperative localization in wireless networks," Proc. IEEE, vol. 97, no. 2, pp. 427-450, Feb. 2009.

[3] J. Lien, U. J. Ferner, W. Srichavengsup, H. Wymeersch, and M. Z. Win, "A comparison of parametric and sample-based message representation in cooperative localization," International Journal of Navigation and Observation, 2012.

[4] M. A. Caceres, F. Penna, H. Wymeersch, and R. Garello, "Hybrid GNSS-terrestrial cooperative positioning via distributed belief propagation," in IEEE Global Telecommun. Conf., Dec. 2010, pp. 1-5.

[5] P.-A. Oikonomou-Filandras and K.-K. Wong, "Hybrid nonparametric belief propagation for localization in wireless networks," in IEEE Sensor Signal Process. Defence, London, UK, Sep. 2011.

[6] M. Welling and J. J. Lim, "A distributed message passing algorithm for sensor localization," in Proc. Int. Conf. Artifical neural networks, Berline, Heidelberg, 2007, pp. 767-775, Springer-Verlag.

[7] K. Das and H. Wymeersch, "Censoring for Bayesian cooperative positioning in dense wireless networks," IEEE J. Sel. Areas Commun., vol. 30, no. 9, pp. 1835-1842, Oct. 2012.

[8] V. Savic, A. Poblacioón, S. Zazo, and M. García, “An experimental study of RSS-based indoor localization using nonparametric belief propagation based on spanning trees," in Int. Conf. on Sensor Technol. and Appl. (SENSORCOMM), July 2010, pp. 238-243.

[9] V. Savic and S. Zazo, "Nonparametric generalized belief propagation based on pseudo-junction tree for cooperative localization in wireless networks," EURASIP Journal on Advances in Signal Processing, vol. 2013, no. 1, pp. 16, 2013.

[10] A. T. Ihler, J. W. Fisher, R. L. Moses, and A. S. Willsky, "Nonparametric belief propagation for self-localization of sensor networks," IEEE J. Sel. Areas Commun., vol. 23, no. 4, pp. 809-819, Apr. 2005.

[11] Christopher M. Bishop, Pattern Recognition and Machine Learning (Information Science and Statistics), SpringerVerlag New York, Inc., Secaucus, NJ, USA, 2006.

[12] D. Jin, F. Yin, C. Fritsche, A. M. Zoubir, and F. Gustafsson, "Efficient cooperative localization algorithm in LOS/NLOS environments," in European Signal Process. Conf., Aug. 2015.

[13] N. Patwari, A. O. Hero, M. Perkins, N. S. Correal, and R. J. O'Dea, "Relative location estimation in wireless sensor networks," IEEE Trans. Signal Process., vol. 51, no. 8, pp. 21372148, Aug 2003. 